\title{
Projeção do diâmetro de árvores de Hevea brasiliensis por meio de modelos mistos não lineares
}

O estudo teve como objetivo avaliar a acurácia de modelos mistos não lineares na projeção do crescimento em diâmetro de árvores individuais de Hevea brasiliensis. A área de estudo está localizada no município de Linhares, Espírito Santo e possui área total de $784 \mathrm{~m}^{2}$. As árvores estão plantadas no espaçamento de 2,0 $\times 2,0 \mathrm{~m}$. As medições do diâmetro a 1,3 m do solo das árvores foram realizadas anualmente dos dois aos 14 anos de idade. Foram ajustados três modelos não lineares considerando efeitos fixos e efeitos aleatórios, sendo estes os modelos de Pienaar e Schiver, Mitscherlich e Chapman-Richards. A avaliação das estimativas geradas pelos modelos mistos e fixos foi realizada, tanto para o ajuste como para a projeção, com base no coeficiente de correlação (r), viés [V (\%)], relative root mean square error [RMSE(\%)]. O desempenho dos modelos de regressão quando considerado também efeitos aleatórios foi superio aos modelos de efeito fixo, sendo capaz de modelar a heterocedasticidade e a autocorrelação observada na análise gráfica dos ajustes dos modelos com efeito fixo. O RMSE mais baixo dos modelos de efeito fixo foi 4,53\% e para o efeito misto foi 3,71\%. Quando comparado o valor de RMSE da projeção, o menor valor obtido com o modelo de efeito fixo foi de $22 \%$ e com efeito misto de 4,38\%. A utilização de modelos de efeitos fixos e aleatórios resultou em ganhos significativos de acurácia, boa aplicação em dados agrupados e permitiu modelar a heterocedasticidade e a autocorrelação dos dados.

\section{Projection of the diameter of Hevea brasiliensis trees by means of nonlinear mixed models}

\begin{abstract}
The objective of this study was to evaluate the accuracy of nonlinear mixed effect regression models in the growth projection of individual trees of Hevea brasiliensis. Planting is in the municipality of Linhares in the state of Espírito Santo and has a total area of $784 \mathrm{~m}^{2}$. The trees are planted at $2.0 \times 2.0 \mathrm{~m}$ spacing. Tree measurements were performed annually from two to 14 years. Three nonlinear models were fitted with fixed and mixed effects, these being the models of Pienaar and Schiver, Mitscherlich and Chapman-Richards. The evaluation of those generated by the mixed and fixed models was performed, both for the adjustment and for the projection, based on the correlation coefficient ( $r$ ), bias [V (\%)], relative root mean square erro [RMSE (\%)] The performance of the mixed effect resresion model was ( 作 improvements in accuracy, good application in pooled data and allowed to model the heteroscedasticity and autocorrelation of the data.
\end{abstract}

Keywords: Growth and production models; Autocorrelation; Heteroscedasticity.

Sandro da Silva Barros (iD

Universidade Federal do Espírito Santo, Brasi http://lattes.cnpq.br/0316971600932023

http://orcid.org/0000-0003-3805-9721 sandrobarros@bol.com.br

Jeferson Pereira Martins Silva (D)

Universidade Federal do Espírito Santo, Brasil http://lattes.cnpq.br/6748966859692740

http://orcid.org/0000-0003-1552-1127

jefersonsb09@hotmail.com

\section{Evandro Ferreira da Silva (iD}

Universidade Federal do Piauí, Brasil

http://lattes.cnpq.br/5248232715871726

http://orcid.org/0000-0001-5948-6402

evandroflorestal@gmail.com

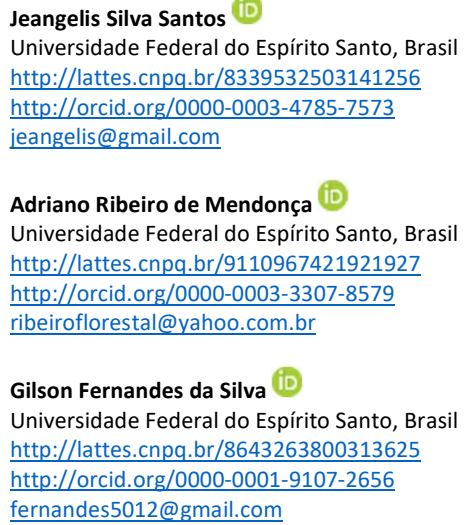

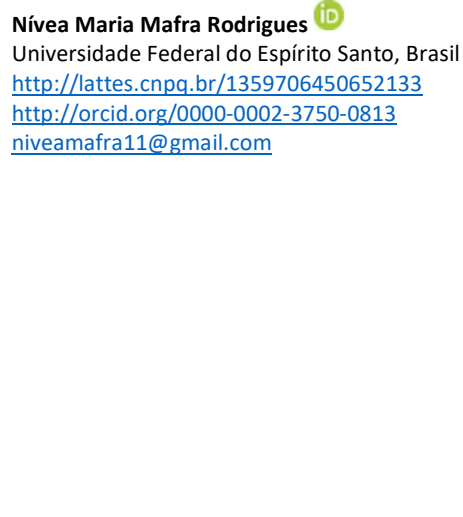

Nívea Maria Mafra Rodrigues

$02-3750-0813$

niveamafra11@gmail.com

\section{Referencing this:}

BARROS, S. S.; SILVA, J. P. M.; SILVA, E. F.; SANTOS, J. S.; MENDONÇA, A. R.; SILVA, G. F.; RODRIGUES, N. M. M.. Projeção do diâmetro de árvores de Hevea brasiliensis por meio de modelos mistos não lineares. Revista Ibero Americana de Ciências Ambientais, v.12, n.1, p.31-41, 2021. DOI: http://doi.org/10.6008/CBPC2179- 


\section{INTRODUÇÃO}

A Hevea brasiliensis [(Willd. ex Adr. de Juss.) Müell. Arg.], conhecida popularmente por seringueira, é nativa da bacia Amazônica (CHEEK, 2002; LORENZI, 2009), e possui vasto potencial econômico com inúmeras utilizações do seu látex na fabricação de diversos produtos (VIEIRA et al., 2010). De acordo com IBÁ (2017), a área plantada com Hevea brasiliensis fica atrás somente de espécies exóticas dos gêneros Eucalyptus e Pinus.

Estudos sobre projeção do diâmetro e padrões de crescimento de árvores individuais são importantes no manejo florestal, uma vez que permite definir a seleção de árvores para exploração e proteção, além de estimar ciclos de corte e prescrever tratamentos silviculturais (ADAME et al., 2008a).

Para um bom planejamento da colheita desses plantios, é necessário prognosticar a produção. Neste contexto, os modelos de crescimento e produção são utilizados para projetar variáveis dendrométricas e simular diferentes alternativas de manejo (LEITES et al., 2009; VANCLAY, 1994).

Os modelos de crescimento em diâmetro e altura são importantes para prever o crescimento (LHOTKA et al., 2011; TIMILSINA et al., 2013) e, consequentemente, são utilizados para obter a produção de variáveis importantes do plantio, que servirão de base para análises econômicas do projeto (HOSOKAWA et al., 1998).

O ajuste de modelos de regressão é o método estatístico mais comumente utilizado na estimação de variáveis dendrométricas (GRÉGOIRE et al., 1995). Para o ajuste desses modelos são necessários dados de monitoramento das variáveis. No caso de árvores individuais, a medição é feita de forma periódica no mesmo indivíduo ao longo do tempo (XU et al., 2014).

Como os dados para modelagem do crescimento individual são medidas repetidas do mesmo indivíduo no tempo, pode ocorrer correlação temporal ou até mesmo espacial, que resulta no não atendimento dos pressupostos da regressão (FOX et al., 2001). A hipótese de erros independentes é, nesses casos, violada, o que acarreta estimativas não cofiáveis. Afim de evitar os problemas citados, outras técnicas podem ser empregadas em substituição às técnicas clássicas de regressão, como modelos mistos. Diversas pesquisas recentes têm apresentados bons resultados usando modelos de regressão não lineares mistos (MNLM) (XU et al., 2014; CALEGARIO et al., 2004; GOUVEIA et al., 2015).

Os modelos mistos não lineares (MNLM) apresentam em sua estrutura os efeitos fixos e aleatórios, além do erro experimental, sendo que os efeitos aleatórios são viáveis para modelar a variabilidade que não pode ser explicada pelas variáveis fixas no modelo de regressão. Com isso, permitem generalizar estruturas de correlações espaço-temporais e variância não constante (CARVALHO et al., 2014). Estes também podem extrair melhor as relações de variáveis independentes com dados agrupados quando comparado a modelos de regressão tradicionais, sendo uma opção atraente para estimação do crescimento e produção florestal (CALEGARIO et al., 2004; GOUVEIA et al., 2015).

Diante do exposto, a presente pesquisa teve como objetivo avaliar a acurácia dos MNLM na projeção do crescimento em diâmetro de árvores de um plantio de Hevea brasiliensis em comparação a modelos de 
regressão clássicos.

\section{MATERIAIS E MÉTODOS}

\section{Descrição da área}

A área analisada consiste de um plantio de Hevea brasiliensis para fins de observação instalado em 1989, em Linhares, estado do Espírito Santo. O plantio seguiu o espaçamento de 2,0 X 2,0 m, sendo a área útil total de $784 \mathrm{~m}^{2}$. Foi medida a circunferência a 1,30 m do solo (CAP) de cada planta a partir do segundo ano e a última medição foi realizada em 2003, quando o plantio tinha 14 anos de idade. Na

Tabela 1 são apresentadas as estatísticas descritivas dos diâmetros de Hevea brasiliensis.

Tabela 1: Estatísticas descritivas dos diâmetros de árvores de um plantio de Hevea brasiliensis.

\begin{tabular}{lllll}
\hline \multirow{2}{*}{ Idade (anos) } & \multicolumn{1}{l}{$D A P(\mathrm{~cm})$} & & Máximo & $C$ (\%) \\
\cline { 2 - 4 } & Mínimo & Média & 6,63 \\
\hline 3 & 5,09 & 5,58 & 8,59 & 10,20 \\
4 & 5,09 & 7,11 & 11,30 & 14,78 \\
6 & 5,41 & 8,25 & 12,73 & 22,01 \\
7 & 5,09 & 8,50 & 13,69 & 25,86 \\
8 & 5,09 & 8,69 & 15,60 & 27,13 \\
9 & 5,09 & 9,72 & 16,87 & 28,44 \\
10 & 5,41 & 10,31 & 19,10 & 31,08 \\
11 & 5,09 & 11,07 & 20,37 & 32,48 \\
12 & 5,09 & 11,37 & 22,92 & 33,72 \\
14 & 5,25 & 12,20 & 24,19 & 34,88 \\
\hline
\end{tabular}

Em que: $D A P=$ diâmetro a 1,3m do solo; $C V$ = coeficiente de variação.

\section{Modelos analisados e estruturas de variância e autocorrelação}

Foram ajustados três modelos não lineares comumente empregados para modelar o crescimento em diâmetro de árvores individuais: Pienaar et al. (1981) (1), Mitscherlich (2) e Chapman-Richards (3).

$$
\begin{aligned}
& Y_{2}=Y_{1} \cdot \exp ^{\left[-\beta_{0} \cdot\left(I_{2}^{\beta_{1}}-I_{1}^{\beta_{1}}\right)\right]} \cdot \varepsilon \\
& Y_{2}=Y_{1}-\beta_{0} \cdot\left(\beta_{1}^{I_{2}}-\beta_{1}^{I_{1}}\right) \cdot \varepsilon \\
& Y_{2}=Y_{1} \cdot\left(\frac{1-\exp ^{\left(\beta_{1} \cdot I_{2}\right)}}{1-\exp ^{\left(\beta_{1} \cdot I_{1}\right)}}\right)^{\beta_{0}} \cdot \varepsilon
\end{aligned}
$$

Em que: $Y_{1}=$ diâmetro $(\mathrm{cm})$ na idade corrente; $Y_{2}=$ diâmetro $(\mathrm{cm})$ na idade futura; $l_{1}=$ idade corrente (meses); $l_{2}=$ Idade futura (meses); $\beta_{i}$ = coeficientes do modelo; e $\varepsilon=$ erro aleatório.

Os modelos de regressão foram ajustados considerando efeitos fixos e na forma mista (efeitos fixos e aleatórios), com o objetivo de verificar se a inclusão do efeito aleatório resultaria em ganhos relacionados a acurácia na estimação do diâmetro futuro de árvores de seringueira. Para isso, foi testada a árvore como efeito aleatório.

Nos modelos de crescimento de árvores individuais, a variância dos diâmetros, geralmente, aumenta com o aumento do diâmetro das árvores. Para corrigir tal efeito, foram analisadas as funções varPower 
(potência de uma covariável) e varldent (variância constante por grupo). Além disso, para corrigir uma eventual autocorrelação entre os dados observados das medidas repetidas no tempo no mesmo indivíduo, foram testadas duas estruturas de autocorrelação: autorregressiva de primeira ordem (AR1) e a de tempo contínuo autorregressiva de primeira ordem (CAR1).

Os três modelos na forma fixa foram ajustados pelo método Gauss Newton por meio do software $\mathrm{R}$ (R CORE TEAM, 2017), empregando-se a função nls. Para ajuste dos modelos não lineares mistos, foi utilizado o algoritmo da máxima verossimilhança restrita, disponibilizado no pacote MNLM (PINHEIRO et al., 2000).

Para o ajuste dos modelos, os dados foram oganizados com informações do inventário pareadas em intervalos de 12 meses, ou seja, IFC1-IFC2, IFC2-IFC3, e assim sucessivamente até os dez anos. As projeções dos diametros das árvores de seringueira foram feitas com a aplicação das equações já ajustadas no banco de dados do primeiro inventário e foram projetados até os 14 anos, para verificar a capacidade dos modelos em projetar os diâmetro em uma idade superior ao do ajuste (validação). Nesse caso, os diâmetros projetados na idade anterior foram considerados como variável independente para projetar o diametro em uma idade futura com intervalo de 12 meses.

\section{Avaliação da acurácia das estimativas}

A avaliação das estimativas geradas pelos modelos mistos e pelos modelos fixos foi realizada, tanto para o ajuste como para a projeção, com base nas seguintes estatísticas: coeficiente de correlação $(r)$ viés [V (\%)], relative root mean square error [RMSE(\%)] (Tabela 2). Além desses critérios, para o ajuste, foram utilizados o critério de informação de Akaike $(A / C)$ e o critério de informação Bayesiano (BIC).

Tabela 2: Estatísticas para avaliar o desempenho dos modelos não lineares fixos e mistos para projetar o diâmetro futuro das árvores de Hevea brasiliensis.

\begin{tabular}{ll}
\hline Estatísticas & Fórmulas \\
\hline AIC & $A I C=-2 \ln (m v)+2 p$ \\
$B I C$ & $B I C=-2 \ln (m v)+p \ln (n)$ \\
& $r_{y \hat{y}}=\frac{\sum_{i=1}^{n}\left(Y_{i}-\bar{Y}\right)\left(\hat{Y}_{i}-\hat{Y}_{m}\right)}{\sqrt{\left[\sum_{i=1}^{n}\left(Y_{i}-\bar{Y}\right)^{2}\right]\left[\sum_{i=1}^{n}\left(\hat{Y}_{i}-\hat{Y}_{m}\right)^{2}\right]}}$ \\
Coeficiente de correlação & $V(\%)=\frac{100}{\sum_{i=1}^{n}} \frac{\sum_{i}-\sum_{i=1}^{n} \hat{Y}_{i}}{n}$ \\
Viés & $R M S E(\%)=\frac{100}{\bar{Y}} \cdot \sqrt{\frac{\sum_{i=1}^{n}\left(Y_{I}-\hat{Y}_{i}\right)^{2}}{n}}$ \\
Relative root mean square error & (6) \\
\hline
\end{tabular}

Em que: $m v$ = função de máxima verossimilhança do modelo; $p=$ número de parâmetros no modelo; $\mathrm{n}=$ número de observações; $Y_{i}=$ variável dependente observada; $\hat{Y}_{i}=$ variável dependente estimada; $\bar{Y}_{i}=$ média da variável dependente observada; $\hat{Y}_{m}=$ média da variável dependente estimada.

De forma a complementar às estatísticas, foram elaborados gráficos relacionando os valores 
observados e estimados pelas técnicas e gráficos de resíduos percentuais apenas para os melhores modelos, selecionados conforme os critérios propostos para avaliar a qualidade de ajuste e prognose dos diâmetros. O erro de cada observação foi calculado em porcentagem (Equação 7).

$$
E(\%)=\frac{Y_{i}-\hat{Y}_{i}}{Y_{i}} 100
$$

Em que: $E(\%)=$ erro de cada observação.

\section{Detecção da presença de heterocedasticidade e da autocorrelação nas estimativas}

Para a deteç̧ão de heterocedasticidade e autocorrelação não há regras estabelecidas, apenas procedimentos para realizar tal inferência. Existem dois tipos de procedimento, o formal, em que se usa testes, e o informal, por análise gráfica (GUJARATI et al., 2011). Para investigar a possível ocorrência de heterocedasticidade e autocorrelação no presente estudo utilizou-se o procedimento informal por meio do método gráfico que consiste em analisar a disposição dos resíduos em percentagem oriundo da equação gerada no processo de ajuste do modelo (CAMPOS et al., 2017).

\section{RESULTADOS}

Os parâmetros estimados dos modelos tradicionais de efeito fixo para projeção do $D A P$, bem como os respectivos erros padrão e testes de significância podem ser observados na

Tabela 3. Nota-se que todos os parâmetros dos modelos foram significativos $(p<0,01)$ pelo teste $t$.

Tabela 3: Estatísticas do ajuste dos modelos na sua forma original para projeção do diâmetro de árvores de Hevea brasiliensis.

\begin{tabular}{llllll}
\hline Modelo & Parâmetro & Estimativa & Erro padrão & tc & Pr $>|\mathrm{tc}|$ \\
\hline \multirow{2}{*}{ Pienaar e Schiver } & $\beta_{0}$ & 3,79198 & 0,06431 & 58,96 & $<0,001$ \\
& $\beta_{1}$ & $-0,4475$ & 0,03146 & $-14,22$ & $<0,001$ \\
\hline \multirow{2}{*}{ Mitscherlich } & $\beta_{0}$ & 18,1479 & 0,30457 & 59,59 & $<0,001$ \\
& $\beta_{1}$ & 0,8755 & 0,005627 & 155,59 & $<0,001$ \\
\hline \multirow{2}{*}{ Chapman-Richards } & $\beta_{0}$ & 0,4427 & 0,02027 & 21,84 & $<0,001$ \\
& $\beta_{1}$ & $-18,9186$ & 0,84316 & $-22,44$ & $<0,001$ \\
\hline
\end{tabular}

Na Tabela 4 encontram-se as estatísticas e medidas de acurácia utilizadas para avaliar a qualidade do ajuste dos modelos tradicionais e de efeito misto para a prognose do DAP. Os ajustes dos modelos fixos e mistos resultaram em estimativas com acurácia e valores de RMSE (\%) menores que 5,30\%. Foi observado ausência de viés $(V<0,03 \%)$ e coeficientes de correlação superiores a 0,99.

Dentre os modelos tradicionais, o modelo 1 obteve maior acurácia no ajuste, apresentando menor RMSE (\%), $V(\%), A I C$ e $B I C$ e maior valor de $r$. Entretanto, os modelos 2 e 3 também podem ser utilizados para estimar o diâmetro futuro de seringueira, pois houve valores relevantes entre as estatísticas dos modelos analisados. 
De modo geral, os modelos mistos obtiveram maior acurácia no ajuste e homogeneidade nos valores das estatísticas empregadas para a avaliação. Apenas os modelos 8 e 9 resultaram em valores mais discrepantes quando comparados aos demais modelos, considerando o RMSE (\%). Em relação às estatísticas das estimativas utilizando MNLM, os ajustes utilizando a função varldent, que tem o objetivo de modelar a variância do DAP ao longo do tempo, foram superiores quando comparadas a função varPower.

Tabela 4: Estatísticas para avaliar o ajuste dos modelos não lineares fixos e mistos para projeção do DAP de árvores de Hevea brasiliensis.

\begin{tabular}{|c|c|c|c|c|c|c|c|c|}
\hline Autor & Modelo & Função de variância & Função de Autocorrelação & RMSE (\%) & $V(\%)$ & $r$ & $A I C$ & $B I C$ \\
\hline \multicolumn{9}{|l|}{ Modelos fixos } \\
\hline Piennar e Schiver & 1 & - & - & 4,53 & 0,22 & 0,9922 & 2348,22 & 2364,52 \\
\hline Mitscherlich & 2 & - & - & 5,29 & $-0,07$ & 0,9913 & 2874,84 & 2891,15 \\
\hline Chapman-Richards & 3 & - & - & 4,57 & 0,24 & 0,9920 & 2384,15 & 2400,47 \\
\hline \multicolumn{9}{|c|}{ Modelo não linear misto com a árvore como efeito aleatório } \\
\hline \multirow{4}{*}{ Piennar e Schiver } & 4 & \multirow{2}{*}{ varPower } & AR1 & 3,73 & $-0,10$ & 0,9945 & 2268,76 & 2306,81 \\
\hline & 5 & & CAR1 & 3,90 & $-0,28$ & 0941 & 2295,35 & 2333,41 \\
\hline & 6 & \multirow{2}{*}{ varldent } & $A R 1$ & 3,73 & $-0,11$ & 0,9945 & 2264,41 & 2307,90 \\
\hline & 7 & & CAR1 & 3,88 & $-0,30$ & 0,9941 & 2289,72 & 2333,21 \\
\hline \multirow{4}{*}{ Mitscherlich } & 8 & \multirow{2}{*}{ varPower } & $A R 1$ & 5,29 & $-0,11$ & 0,9915 & 2642,72 & 2680,77 \\
\hline & 9 & & CAR1 & 5,29 & $-0,11$ & 0,9915 & 2642,72 & 2680,77 \\
\hline & 10 & \multirow{2}{*}{ varldent } & $A R 1$ & 3,71 & $-0,03$ & 0,9946 & 2191,85 & 2235,34 \\
\hline & 11 & & CAR1 & 3,74 & $-0,03$ & 0,9945 & 2246,15 & 2289,64 \\
\hline \multirow{4}{*}{ Chapman-Richards } & 12 & \multirow{2}{*}{ varPower } & $A R 1$ & 3,88 & 0,07 & 0,9940 & 2163,57 & 2207,05 \\
\hline & 13 & & CAR1 & 3,94 & 0,12 & 0,9939 & 2223,11 & 2266,60 \\
\hline & 14 & \multirow{2}{*}{ varldent } & $A R 1$ & 3,88 & 0,07 & 0,9940 & 2163,85 & 2201,90 \\
\hline & 15 & & CAR1 & 3,94 & 0,12 & 0,9939 & 2221,11 & 2259,16 \\
\hline
\end{tabular}

Em que: $A R 1$ = estrutura autorregressiva de ordem 1; CAR1 = estrutura de tempo contínuo autorregressiva de ordem 1.

As medidas de acurácia para avaliar a projeção do DAP (validação) pelos modelos ajustados encontram-se na Erro! Fonte de referência não encontrada..

Tabela 4: Estatísticas para avaliar a qualidade da projeção do DAP de árvores Hevea brasiliensis com modelos mistos e modelos tradicionais.

\begin{tabular}{|c|c|c|c|c|c|c|}
\hline Autor & Modelo & var_cov & Autocorr & RMSE (\%) & $\mathrm{V}(\%)$ & $r$ \\
\hline \multicolumn{7}{|l|}{ Ajuste tradicional } \\
\hline Piennar e Schiver & 1 & - & - & 22,00 & 1,40 & 0,7866 \\
\hline Mitscherlich & 2 & - & - & 24,09 & 0,48 & 0,7490 \\
\hline Chapman-Richards & 3 & - & - & 23,52 & $-0,20$ & 0,7648 \\
\hline \multicolumn{7}{|c|}{ Modelo não-linear misto com a árvore como efeito aleatório } \\
\hline \multirow{4}{*}{ Piennar e Schiver } & 4 & \multirow{2}{*}{ varPower } & AR1 & 6,82 & 1,72 & 0,9826 \\
\hline & 5 & & CAR1 & 9,36 & 1,00 & 0,9662 \\
\hline & 6 & \multirow{2}{*}{ varldent } & AR1 & 6,82 & 1,73 & 0,9826 \\
\hline & 7 & & CAR1 & 8,83 & 0,75 & 0,9702 \\
\hline \multirow{4}{*}{ Mitscherlich } & 8 & \multirow{2}{*}{ varPower } & AR1 & 23,99 & 0,72 & 0,7525 \\
\hline & 9 & & CAR1 & 23,99 & 0,72 & 0,7525 \\
\hline & 10 & \multirow{2}{*}{ varldent } & AR1 & 4,38 & 0,06 & 0,9927 \\
\hline & 11 & & CAR1 & 4,93 & 0,19 & 0,9916 \\
\hline \multirow{4}{*}{ Chapman-Richards } & 12 & \multirow{2}{*}{ varPower } & AR1 & 7,95 & 0,17 & 0,9770 \\
\hline & 13 & & CAR1 & 8,97 & 0,97 & 0,9709 \\
\hline & 14 & \multirow{2}{*}{ varldent } & AR1 & 7,60 & 0,37 & 0,9790 \\
\hline & 15 & & CAR1 & 8,98 & 0,96 & 0,9708 \\
\hline
\end{tabular}

Em que: $A R 1$ = estrutura autorregressiva de primeira ordem 1; CAR1 = estrutura de tempo contínuo autorregressiva de ordem 1.

O RMSE (\%) dos modelos de efeito fixo resultaram em valores quatro vezes maiores do que no ajuste. Consequentemente, apesar do erro relativamente baixo no ajuste, a aplicação desses modelos para árvores com idades maiores que as que pertenceram a base de dados de ajuste, na prática, iria implicar em 
estimativas de menor acurácia. É possível observar que os modelos mistos tiveram valores de RMSE (\%) superiores aos do ajuste, mas com valores inferiores a 10\%, com exceção do modelo de Mitscherlich utilizando a função varPower, que apresentou erro semelhante aos modelos tradicionais.

Dentre os modelos tradicionais, o modelo de Pienaar e Schiver apresentou desempenho superior aos demais, assim como no ajuste. Entre os modelos mistos, o de Mitscherlich com função varldent obteve os melhores resultados. Ao observar o viés, ocorreu o contrário que no ajuste, sendo quase todos os valores positivos, com exceção do modelo tradicional de Chapman-Richards. O coeficiente de correlação também seguiu a mesma tendência do RMSE (\%), em que os modelos fixos e misto de Mitscherlich com função varPower obtiveram desempenhos inferiores que os demais.

Ao observar os gráficos das relações entre o DAP observado e estimado (Figura 1), fica evidente que os modelos tradicionais (1, 2 e 3 ) apresentaram maior dispersão em relação à linha ideal (1:1), o que representa menor acurácia. Nos melhores modelos mistos (4, 10 e 14), os pontos se apresentaram de forma mais agregada e uniforme, com destaque para o modelo 10.
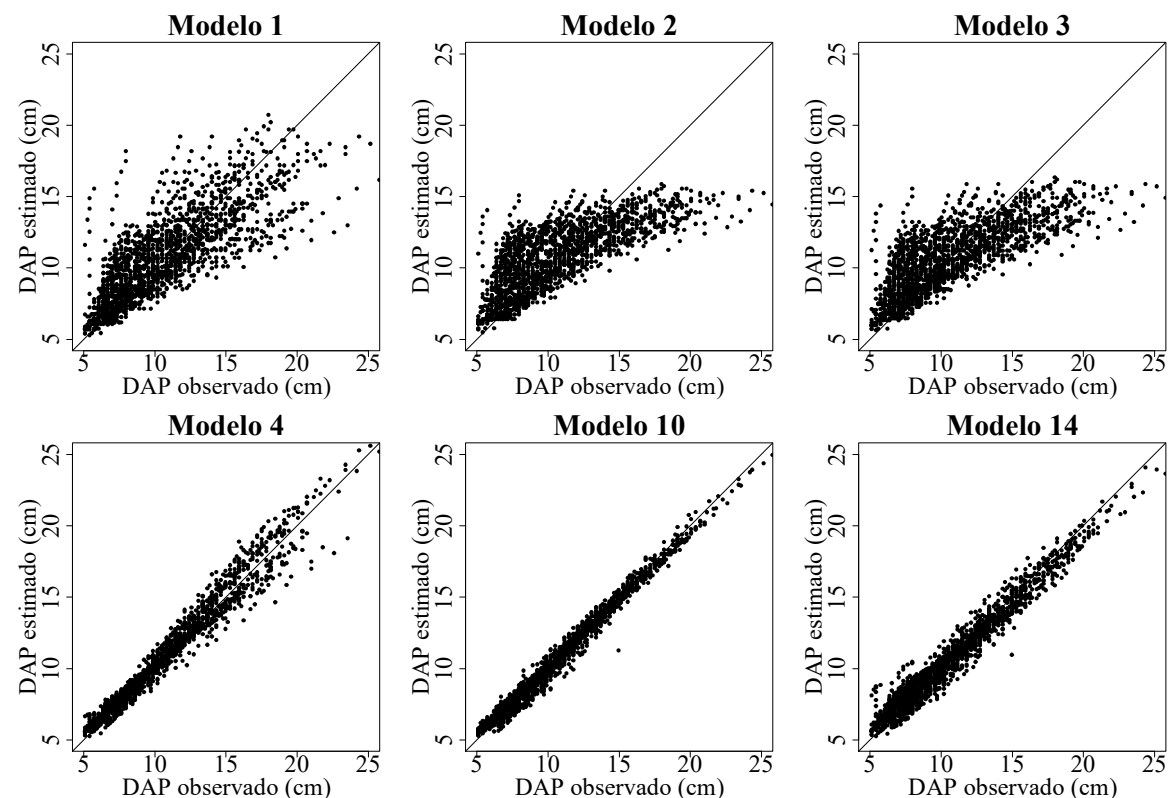

Figura 1: Gráficos de relação entre o DAP estimado e o observado para projeção do diâmetro de árvores de Hevea brasiliensis.
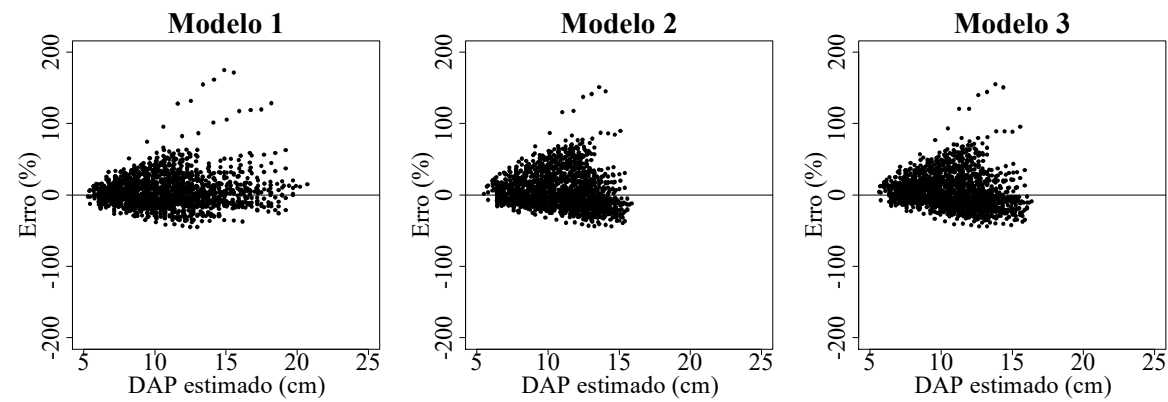

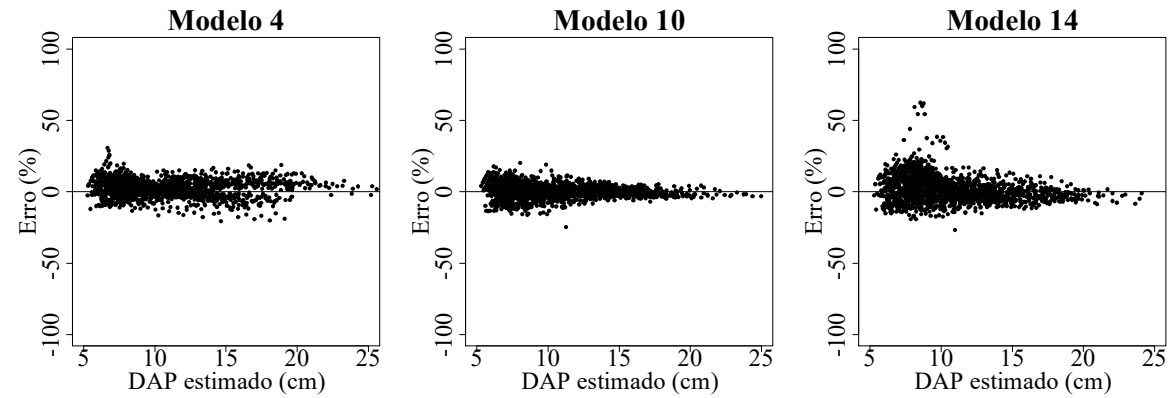

Figura 2: Gráficos de dispersão residual para projeção de diâmetros de árvores de Hevea brasiliensis.

Na Figura 2 encontra-se a dispersão dos erros percentuais em relação ao DAP estimado. É possível notar a mesma tendência de maior dispersão dos erros nos modelos fixos em comparação com os modelos mistos. Além disso, nos modelos fixos ocorre uma tendência de aumentar os erros com o aumento do diâmetro estimado e o inverso ocorreu nos modelos mistos. A análise dos gráficos das Figuras 1 e 2 evidenciam mais uma vez a superioridade do modelo 10 em relação aos demais.

\section{DISCUSSÃO}

Neste trabalho foi possível observar a heterocedasticidade nas estimativas de diâmetro nos modelos 1, 2 e 3 (Figura 2). A disposição dos resíduos apresenta-se com um padrão sistêmico indicando a possível presença de heterocedasticidade (GUJARATI et al., 2011). Na Figura 1, também é possível observar clara tendência ao confrontar os valores observados e estimados. É evidente o aumento da variância com o aumento do diâmetro estimado nos modelos 1, 2 e 3. Isso corrobora com a hipótese de haver necessidade de modelar a heterogeneidade de variância para estes modelos.

As funções de variância usadas neste trabalho (varPower e varlden), permitem que a variância dentro do grupo (árvores) dependa dos efeitos fixos e dos efeitos aleatórios. Tais funções modelam a heterocedasticidade dos erros aleatórios, dando menor peso às observações com grande variância, por exemplo, se a variância cresce ou decresce com determinada covariável (PINHEIRO et al., 2000).

Nas Figuras 1, 2 e 3 é perceptível alguns padrões sistemáticos plausíveis de uma eventual presença de autocorrelação. Nos modelos de projeção, como os avaliados neste trabalho, verifica-se que o $D A P$ atual depende, além de outras variáveis (idade, sitio, índice de competição), do DAP do período anterior. Nestes modelos, a variável independente é o valor defasado da variável dependente e caso isso não seja levado em consideração, o termo do erro seguirá um padrão sistemático decorrente do DAP do período anterior sobre o DAP atual. As estruturas $A R 1$ e CAR1 são úteis para controlar a autocorrelação de dados com medidas repetidas ao longo do tempo e tem trabalhos que já foram feitos usando estes processos, como em Weiskittel et al. (2009) e Gouveia et al. (2015).

Com relação as estruturas de variância e autocorrelação dos modelos utilizados neste trabalho, para modelar a heterocedasticidade e a autocorrelação, não foi verificado diferenças significativas nos indicadores estatísticos. Resultados similares utilizando essas estruturas foram encontrados por Gouveia et al. (2015).

Ao analisar a inclusão do efeito aleatório árvore nos modelos tradicionalmente empregados para a projeção do DAP de árvores de Hevea brasiliensis, os autores assumiram que haveria ganhos de acurácia. 
Pelas medidas de acurácia (Tabelas 4 e 5), nota-se que os modelos MNLM ajustados com a função de variância varPower e varlden e estrutura de correlação corAR1 e corCAR1 apresentaram estimativas mais acuradas do diâmetro projetado, quando comparado aos modelos tradicionais ajustados apenas com efeito fixo. O ganho em termo de acurácia entre o melhor modelo misto e o melhor modelo fixo, para a projeção do diâmetro, com base no RMSE (\%), foi de aproximadamente $80 \%$. Já o modelo de Mitscherlich com a função de varPower (modelos 8 e 9) apresentaram RMSE (\%) semelhante do modelo com efeito fixo.

O ganho de acurácia e também a possibilidade de obter uma redução na quantidade de dados utilizados para ajuste dos modelos foram verificados por outros autores em estudos sobre afilamento de troncos (ÖZÇELIK et al., 2010; MÔRA, 2015), relação hipsométrica (ADAME et al., 2008b; ÖZÇELIK et al., 2013; SHARMA et al., 2016), volume de árvore individual (VISMARA et al., 2016), crescimento e produção (CALEGARIO et al., 2004), mortalidade (GROOM et al., 2012) e tamanho da copa para árvores individuais (FU et al., 2013).

Mendonça et al. (2015) obtiveram ganho de 53,89\% em acurácia com a correção da heterocedasticidade em modelos hipsométricos, Carvalho et al. (2011) tiveram uma redução no erro padrão residual (Syx) de 26,31\% para 4,01\% em predição volumétrica. Já Gouveia et al. (2015), também trabalhando com modelos volumétricos, obtiveram uma redução no erro padrão residual de 26 vezes. Recentemente, Dantas et al. (2020) utilizando modelos volumétricos não linear de efeito fixo e misto reduziram o RMSE (\%) de $18,58 \%$ para 4,42\%, indicando a eficiência do MNLM em relação ao MNL, pois a inclusão de variáveis aleatórias proporcionou melhor desempenho ao modelo, além de conseguir reduzir o erro padrão residual em 53 vezes.

No setor florestal decisões importantes relacionadas ao manejo florestal são norteadas a partir de projeções de crescimento e produção (CAMPOS et al.,2017). A capacidade da utilização de MNLM busca modelar a heterocedasticidade e a autocorrelação encontradas nos dados, por meio de efeitos aleatórios, o que torna uma técnica viável para realiza projeções de diâmetro futuro de espécies florestais.

Cabe ressaltar, que o diâmetro projetado foi em idades superiores ao ajuste dos modelos, o que representa uma informação importante para o planejamento florestal. O que corrobora a vantagem de aplicação da técnica em dados com medidas repetidas no tempo.

Na modelagem do DAP futuro de árvores de Hevea brasiliensis, utilizando os modelos fixos clássicos, verificou-se a necessidade de se incluir efeitos aleatórios. Associado à inclusão dos efeitos aleatórios, a modelagem da heterogeneidade da variância e da autocorrelação melhorou significativamente os indicadores estatísticos e as análises gráficas.

\section{CONCLUSÕES}

A utilização de modelos mistos não lineares promoveu ganho de acurácia quando comparado com os modelos não lineares tradicionais de efeito fixo. Dentre os modelos avaliados, o que obteve o melhor desempenho foi o que utilizou a função varldent para modelar os efeitos da variância e com estrutura autorregressiva de primeira ordem para modelar a autocorrelação entre os dados observados das medidas 
repetidas no tempo.

\section{REFERÊNCIAS}

ADAME, P.; HYNYNEN, J.; CAÑELLAS, I.; DEL RÍO, M. Individual-tree diameter growth model for rebollo oak (Quercus pyrenaica Willd.) coppices. Forest Ecology and Management, v.255, n.3-4, p.1011-1022, 2008a. DOI: https://doi.org/10.1016/i.foreco.2007.10.019

ADAME, P.; DEL RÍO, M.; CAÑELLAS, I.. A mixed nonlinear height-diameter model for pyrenean oak (Quercus pyrenaica Willd.). Forest Ecology and Management, v.256, n.1-2, p.88-98, 2008b. DOI:

https://doi.org/10.1016/j.foreco.2008.04.006

CALEGARIO, N.; DANIELS, R. F.; MAESTRI, R.; NEIVA, R.. Desenvolvimento de um modelo linear de efeito misto na estimativa do crescimento e produção de povoamentos clonais. Cerne, Lavras, v.10, n.1, p.67-86, 2004.

CAMPOS, J. C. C.; LEITE, H. G.. Mensuração florestal: perguntas e respostas. 5 ed. Viçosa: UFV, 2017.

CARVALHO, S. P. C.; RODRIGUEZ, L. C. E.; CALEGARIO, N.; SAVIAN, T. V.; LIMA, M. P.; SILVA, C. A.; MENDONÇA, A. R.; NICOLETTI, M. F.. Modelagem não linear mista para descrever o afilamento de árvores clonais de Eucalyptus sp. Scientia Forestalis, Piracicaba, v.42, n.104, p.605-614, 2014.

CARVALHO, S. P. C.; CALEGARIO, N.; SILVA, F. F.; BORGES, L. A. C.; MENDONÇA, A. R.; LIMA, M. P.. Modelos não lineares generalizados aplicados na predição da área basal e volume de Eucalyptus clonal. Cerne, v.17, n.4, p.541-548, 2011. DOI: http://dx.doi.org/10.1590/S0104-77602011000400013

CHEEK, M.. Flora da Reserva Ducke: guia de identificação das plantas vasculares de uma floresta de terra-firme na Amazônia Central. Kew Bulletin, v.57, n.1, p.238, 2002.

DANTAS, D.; CALEGARIO, N.; ACERBI, F. W.; CARVALHO, S. P. C.; ISAAC JÚNIOR, M. A.; MELO, E. A.. Multilevel nonlinear mixed-effects model and machine learning for predicting the volume of Eucalyptus spp. trees. Cerne, Lavras, v.26, n.1, p.48-57, 2020. DOI:

https://doi.org/10.1590/01047760202026012668

FOX, J. C.; ADES, P. K.; BI, H.. Stochastic structure and individual-tree growth models. Forest Ecology and Management, v.1, n.1-2, p.261-276, 2001. DOI: https://doi.org/10.1016/S0378-1127(00)00632-0

FU, L.; SUN, H.; SHARMA, R. P.; LEI, Y.; ZHANG, H.; TANG, S.. Nonlinear mixed-effects crown width models for individual trees of Chinese fir (Cunninghamia lanceolata) in southcentral China. Forest Ecology and Management, v.302, p.210-220, 2013. DOI: https://doi.org/10.1016/i.foreco.2013.03.036

GOUVEIA, J. F.; SILVA, J. A. A.; FERREIRA, R. L. C.; GADELHA, F. H. L.; LIMA FILHO, L. M. A.. Modelos volumétricos mistos em clones de Eucalyptus no polo gesseiro do Araripe, Pernambuco. Floresta, Curitiba, v.45, n.3, p.587-598, 2015. DOI: https://doi.org/10.5380/rf.v45i3.36844

GRÉGOIRE, T. G.; SCHABENBERGER, O.; BARRETT, J. P.. Linear modelling of irregularly spaced, unbalanced, longitudinal data from permanent-plot measurements. Canadian Journal of Forest Research, Ottawa, v.25, p.137-156, 1995. DOI: https://doi.org/10.1139/x95-017

GROOM, J. D.; HANN, D. W.; TEMESGEN, H.. Evaluation of mixed-effects models for predicting Douglas-fir mortality. Forest Ecology and Management, v.276, p.139-145, 2012. DOI: https://doi.org/10.1016/j.foreco.2012.03.029

GUJARATI, D. N.; PORTER, D. C.. Econometria Básica. 5 ed. Porto Alegre: AMGH, 2011.

HOSOKAWA, R. T.; MOURA, J. B.; CUNHA, U. S.. Introdução ao manejo e economia de florestas. Curitiba: UFPR, 1998.

IBÁ. Indústria Brasileira de Árvores. Relatório 2017: ano base 2016. Brasília: IBÁ, 2017.

LEITES, L. P.; ROBINSON, A. P.; CROOKSTON, N. L.. Accuracy and equivalence testing of crown ratio models and assessment of their impact on diameter growth and basal area increment predictions of two variants of the Forest Vegetation Simulator. Canadian Journal of Forest Research, Ottawa, v.39, n.3, p.655-665, 2009. DOI: https://doi.org/10.1139/X08-205

LHOTKA, J. M.; LOEWENSTEIN, E. F.. An individual-tree diameter growth model for managed uneven-aged oakshortleaf pine stands in the Ozark Highlands of Missouri, USA. Forest Ecology and Management, v.261, n.3, p.770778, 2011. DOI:

https://doi.org/10.1016/i.foreco.2010.12.008

LORENZI, H.. Árvores brasileiras: manual de identificação e cultivo de plantas arbóreas nativas do Brasil - III. 3 ed. Nova Odessa: Instituto Plantarum, 2009.

MENDONÇA, A, R.; CARVALHO, S. P. C.; CALEGARIO, N. Modelos hipsométricos generalizados mistos na predição da altura de Eucalyptus sp. Cerne, Lavras, v.21, n.1, p.107-115, 2015. DOI:

https://doi.org/10.1590/01047760201521011191

MÔRA, R.. Funções de afilamento de forma variável e modelagem de efeitos mistos em fustes de Pinus taeda e Eucalyptus saligna. Tese (Doutorado em Engenharia Florestal) - Universidade Federal do Paraná, Curitiba, 2015.

ÖZÇELIK, R.; DIAMANTOPOULOU, M. J.; CRECENTE-CAMPO, F.; ELER, U.. Estimating Crimean juniper tree height using nonlinear regression and artificial neural network models. Forest Ecology and Management, v.306, p.52-60, 2013. DOI: https://doi.org/10.1016/i.foreco.2013.06.009

ÖZÇELIK, R.; BROOKS, J. R.; JIANG, L.. Modeling stem profile of Lebanon cedar, Brutian pine, and Cilicica fir in Southern Turkey using nonlinear mixed-effects models. European Journal of Forest Research, v.130, n.4, p.613-621, 2010. DOI: https://doi.org/10.1007/s10342-010-0453-5

PINHEIRO, J. C.; BATES D. M.. Mixed-effects models in S and 
S-PLUS. New York: Springer-Verlag, 2000.

R CORE TEAM. R: a language and environment for statistical computing. Viena: R Foundation for Statistical Computing, 2017.

SHARMA, R. P.; VACEK, Z.; VACEK, S.. Nonlinear mixed effect height-diameter model for mixed species forests in the central part of the Czech Republic. Journal of Forest Science, v.62, p.470-484, 2016. DOI:

https://doi.org/10.17221/41/2016-JFS

TIMILSINA, N.; STAUDHAMMER, C. L.. Individual tree-based diameter growth model of slash pine in Florida using nonlinear mixed modeling. Forest Science, v.59, n.1, p.2737, 2013. DOI: https://doi.org/10.5849/forsci.10-028

VANCLAY, J. K.. Modelling forest growth and yield: applications to mixed tropical forests. London: $\mathrm{CAB}$ international, 1994.

VIEIRA, M. R.; GOMES, E. C.; SIVA, H. A. S.. Redução na produção de látex da seringueira provocada pela infestação de ácaros. Revista Ceres, Viçosa, v.57, n.5, p.608613, 2010. DOI: http://dx.doi.org/10.1590/S0034-737X2010000500007

VISMARA, E. S.; MEHTÄTALO, L.; BATISTA, J. L. F.. Linear mixed-effects models and calibration applied to volume models in two rotations of Eucalyptus grandis plantations. Canadian Journal of Forest Research, v.46, n.1, p.132-141, 2016. DOI: https://doi.org/10.1139/cjfr-2014-0435

WEISKITTEL, A. R.; HANN, D. W.; HIBBS, D. E.; LAM, T. Y.; BLUHM, A. A.. Modeling top height growth of red alder plantations. Forest Ecology and Management, Amsterdam, v.258, n.3, p.323-331, 2009. DOI:

http://doi.org/10.1016/j.foreco.2009.04.029

XU, H.; SUN, Y.; WANG, X.; FU, Y.; DONG, Y.; LI, Y.. Nonlinear mixed-effects (NLME) diameter growth models for individual China-fir (Cunninghamia lanceolata) trees in southeast China. PLoS ONE, v.9, n.8, p. e104012, 2014. DOI: https://doi.org/10.1371/journal.pone.0104012

A CBPC - Companhia Brasileira de Produção Científica (CNPJ: 11.221.422/0001-03) detém os direitos materiais desta publicação. Os direitos referem-se à publicação do trabalho em qualquer parte do mundo, incluindo os direitos às renovaç̃oes, expansões e disseminações da contribuiç̃o, bem como outros direitos subsidiários. Todos os trabalhos publicados eletronicamente poderão posteriormente ser publicados em coletâneas impressas sob coordenação da Sustenere Publishing, da Companhia Brasileira de Produção Científica e seus parceiros autorizados. Os (as) autores (as) preservam os direitos autorais, mas não têm permissão para a publicação da contribuição em outro meio, impresso ou digital, em português ou em tradução. 